\title{
Use of Cardiac Cycle Locating to Minimize the Influence of Cardiac Artifacts on Esophageal Pressure Measurement During Dynamic Occlusion Test
}

\author{
Xuan He MSc, Xiu-Mei Sun MSc, Guang-Qiang Chen MD, Yan-Lin Yang MD, \\ Zhong-Hua Shi MD, Ming Xu MD, and Jian-Xin Zhou MD
}

\begin{abstract}
BACKGROUND: The dynamic occlusion test is used to guide balloon catheter placement during esophageal pressure $\left(P_{\mathrm{es}}\right)$ monitoring. We introduced a cardiac cycle locating method to attenuate the influence of cardiac artifacts on $P_{e s}$ measurement. The aim was to provide a reliable analytic algorithm for the occlusion test. METHODS: Esophageal balloon catheters were placed in subjects receiving pressure support ventilation. During balloon position adjustment, end-expiratory occlusion was performed to induce 3 consecutive inspiratory efforts. $P_{e s}$ and airway pressure $\left(P_{\text {aw }}\right)$ data were collected for off-line analysis. For each occluded inspiratory effort, the change in $P_{e s}\left(\Delta P_{e s}\right)$ was plotted against the change in $P_{a w}\left(\Delta P_{a w}\right)$, and the slope of the regression line was calculated. The $\Delta \mathbf{P}_{\text {es }} / \Delta \mathbf{P}_{\text {aw }}$ ratio was also measured with the cardiac cycle locating method and peak-to-peak method. Bland-Altman analysis was used to assess the agreement between the $\Delta \mathbf{P}_{\text {es }} / \Delta \mathbf{P}_{\text {aw }}$ ratio and the slope. We defined the occlusion test with all fitted slopes for the 3 inspiratory efforts within 0.8 to 1.2 to indicate optimal balloon position; otherwise, the position was deemed non-optimal. Using the slope as the reference, the diagnostic accuracy of the $\Delta P_{e s} / \Delta P_{a w}$ ratio in distinguishing the optimal and the non-optimal balloon position was analyzed. RESULTS: A total of 86 occlusion tests containing 258 inspiratory efforts were collected from 15 subjects. The median (interquartile range) slope of $\Delta P_{\text {es }}$ versus $\Delta P_{\text {aw }}$ plot was $0.85(0.76,0.91)$. Bias (lower and upper limit of agreement) of $\Delta \mathrm{P}_{\mathrm{es}} / \Delta \mathrm{P}_{\mathrm{aw}}$ ratio measured by the cardiac cycle locating method and the peak-to-peak method was $0.02(-0.13$ to 0.16$)$ and $0.06(-0.18$ to 0.31$)$, respectively. Forty-five $(52.3 \%)$ occlusion tests indicated optimal balloon positions. Compared to the peak-to-peak method, the cardiac cycle locating method was more specific in detecting the non-optimal position. CONCLUSIONS: The cardiac cycle locating method provided reliable and precise measurement for the occlusion test. This method can accurately detect non-optimal balloon position during catheter adjustment. Key words: esophageal pressure; occlusion test; cardiac artifact; measurement. [Respir Care 2018;63(2):169-176. (C) 2018 Daedalus Enterprises]
\end{abstract}

\section{Introduction}

Esophageal pressure $\left(\mathrm{P}_{\mathrm{es}}\right)$, applied as a surrogate for pleural pressure, is commonly measured via catheter with

All authors are affiliated with the Department of Critical Care Medicine, Beijing Tiantan Hospital, Capital Medical University, Beijing, China. Dr. Yan-Lin Yang is also affiliated with the Intensive Care Unit, Beijing Electric Power Hospital, Capital Medical University, Beijing, China.

Supplementary material related to this paper is available at http:// www.rcjournal.com. an air-filled balloon placed in the lower two thirds of the esophagus. ${ }^{1,2}$ Since first introduced in 1982, Baydur's occlusion test has been widely used to guide the esophageal

\footnotetext{
The authors have disclosed no conflicts of interest.

Correspondence: Jian-Xin Zhou MD, Department of Critical Care Medicine, Beijing Tiantan Hospital, Capital Medical University, No. 6, Tiantan Xili, Dongcheng District, 100050, Beijing, China. E-mail: zhoujx.cn@icloud.com.
}

DOI: $10.4187 /$ respcare. 05750 
balloon positioning for patients breathing spontaneously. ${ }^{3}$ After the balloon is placed in the esophagus, inspiratory effort during airway occlusion induces simultaneously decreasing in airway pressure $\left(\mathrm{P}_{\mathrm{aw}}\right)$ and $\mathrm{P}_{\mathrm{es}}$. A ratio between negative deflections in $\mathrm{P}_{\mathrm{es}}$ and $\mathrm{P}_{\mathrm{aw}}\left(\Delta \mathrm{P}_{\mathrm{es}} / \Delta \mathrm{P}_{\mathrm{aw}}\right.$ ratio) close to unity indicates a valid surrogate of $\mathrm{P}_{\mathrm{es}}$ for pleural pressure. ${ }^{1-3}$ Conventionally, the $\Delta \mathrm{P}_{\mathrm{es}} / \Delta \mathrm{P}_{\mathrm{aw}}$ ratio was measured with the peak-to-peak method (ie, the pressure change was computed using the amplitude of the peak positive value before the onset of inspiratory effort and the peak negative value at the maximal effort). ${ }^{3,4}$ However, cardiac artifacts on $\mathrm{P}_{\mathrm{es}}$ tracings may affect the accuracy of measurement. . $^{3-6}$ Previous studies have found that oscillations of $\mathrm{P}_{\mathrm{es}}$ due to cardiac pulsations were as high as $3 \mathrm{~cm} \mathrm{H}_{2} \mathrm{O},{ }^{4,7}$ Although signal filtering was attempted to remove the cardiac artifacts, this technique was only suitable for regular $\mathrm{P}_{\mathrm{es}}$ signals during tidal breaths. ${ }^{8-10}$ As for the analysis of occlusion test, signal filtering was relatively sophisticated and subject to unavoidable information loss.

In our study, we introduce a method for $\mathrm{P}_{\mathrm{es}}$ measurement based on cardiac cycle locating to attenuate the influence of cardiac artifacts on the calculation of the $\Delta \mathrm{P}_{\mathrm{es}} / \Delta \mathrm{P}_{\mathrm{aw}}$ ratio during a dynamic occlusion test. The reliability and precision of this method were compared with those of the conventional peak-to-peak method. The accuracy of the introduced method in distinguishing between the optimal and non-optimal balloon position was also analyzed to determine whether we could devise a reliable analytic algorithm for the occlusion test.

\section{Methods}

\section{Study Setting, Ethics, and Population}

This prospective observational study was conducted in the ICU of Beijing Tiantan Hospital, Capital Medical University, Beijing, China. The study protocol was reviewed and approved by the local institutional review board (KY2016-019-02). Written informed consent was obtained from subjects or appropriate substitute decision makers. This study was supported by grants from Special Program of Beijing Municipal Science and Technology Commission (Z161100000116081). The sponsor had no role in the study design, data collection, data analysis, data interpretation, or writing of the report.

In our institute, $\mathrm{P}_{\mathrm{es}}$ monitoring is routinely used in patients with difficult weaning to measure the pressure-time product and to detect patient-ventilator asynchrony. In our study, intubated and mechanically ventilated subjects who failed initial weaning process were included. Exclusion criteria were age $<18 \mathrm{y}$; evidence of arrhythmia; diagnosed or suspected esophageal varices; history of esophageal, gastric, or lung surgery; evidence of active air leak from the lung, including bronchopleural fistula, pneumo-

\section{QUICK LOOK}

\section{Current knowledge}

Esophageal pressure can be used as a surrogate for pleural pressure in mechanically ventilated subjects, and catheters with air-filled balloons are commonly used. The dynamic occlusion test has been widely accepted for the confirmation of balloon position. However, cardiac artifacts on the esophageal pressure tracing may influence the measurement.

\section{What this paper contributes to our knowledge}

A cardiac cycle locating method was introduced to provide reliable and precise measurement for the occlusion test. This method accurately detected non-optimal balloon position during balloon adjustment, and thus may decrease the probability of false optimal balloon placement.

thorax, pneumomediastinum, or an existing chest tube; and evidence of severe coagulopathy.

\section{Esophageal Catheter Placement and Dynamic Occlusion Test}

During the procedure, subjects remained in a supine position, ventilated by a Servo-i ventilator (Maquet, Solna, Sweden) with pressure support mode set at 5-10 $\mathrm{cm} \mathrm{H}_{2} \mathrm{O}$, PEEP 5-10 $\mathrm{cm} \mathrm{H}_{2} \mathrm{O}$, and $\mathrm{F}_{\mathrm{IO}_{2}} 0.35-0.45$.

We used a commercially available esophageal balloon catheter (Cooper Surgical, Trumbull, Connecticut), 5 Fr in diameter and $85 \mathrm{~cm}$ in length, enclosed with a polyethylene balloon. The balloon inflating volume is recommended as $1.0 \mathrm{~mL}$ by the manufacturer. ${ }^{11}$

Two KT 100D-2 pressure transducers (KleisTEK Engineering) were connected proximally to the endotracheal tube to measure the $\mathrm{P}_{\mathrm{aw}}$ and the balloon lumen of the esophageal catheter to measure the $\mathrm{P}_{\mathrm{es}}$, respectively. A heated Fleisch pneumotachograph (Vitalograph, Lenexa, Kansas) was placed between the Y-piece of the ventilator circuit and the endotracheal tube to measure the flow. Pressure transducers and the Fleisch pneumotachograph were connected to an ICU-Lab pressure box (KleisTEK Engineering) with $80-\mathrm{cm}$ rigid tube lines. Before each subject's monitoring, the pressure transducer was calibrated with a water column, and the pneumotachograph with a 1-L calibration syringe (Hans Rudolph Shawnee, Kansas). Signals were displayed continuously and saved (ICU-Lab 2.5 Software Package, KleisTEK Engineering) in a laptop for further analysis, at a sample rate of $200 \mathrm{~Hz}$.

After anesthetizing the nostrils and oropharynx with 10\% lidocaine spray, the catheter was inserted through the nos- 

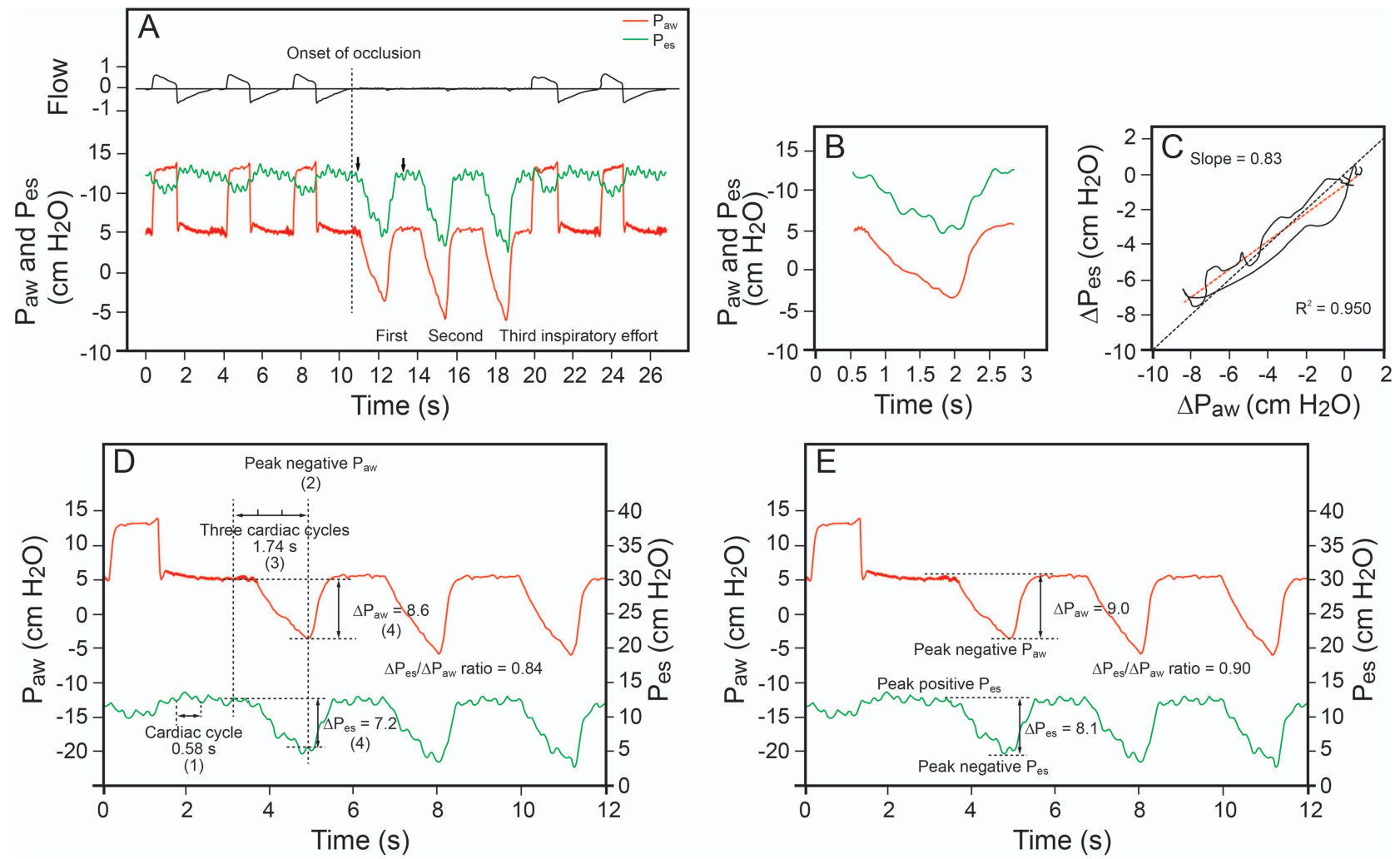

Fig. 1. Example epoch containing flow, airway pressure $\left(P_{\text {aw }}\right)$ and esophageal pressure $\left(P_{\text {es }}\right)$ of the 3 inspiratory efforts during an occlusion test. A: The first, the second, and the third inspiratory effort are indicated. The point of flow returning to zero is marked as the onset of occlusion (dashed line). The start and the end of an inspiratory effort are identified at the same stage of cardiac pulsation (arrows). $B$ : $P_{\text {aw }}$ and $\mathrm{P}_{\text {es }}$ data within the first inspiratory effort are used in linear regression. $\mathrm{C}: \Delta \mathrm{P}_{\text {es }}$ is plotted against $\Delta \mathrm{P}_{\text {aw }}$, and the slope is calculated (red dashed line). Unity is shown as black dashed line. D: The cardiac cycle locating method is performed at 4 steps: (1) length of cardiac cycle is measured by rhythmic pulsation in the $\mathrm{P}_{\text {es }}$ tracing; (2) time point of the peak negative $\mathrm{P}_{\mathrm{aw}}$ during the occluded inspiratory effort is confirmed, and $\mathrm{P}_{\mathrm{aw}}$ and $\mathrm{P}_{\text {es }}$ values are measured; (3) baseline $\mathrm{P}_{\mathrm{aw}}$ before the inspiratory effort is localized and measured at the point with integers of cardiac cycle prior to the peak negative $\mathrm{P}_{\text {aw }}$ point, and $\mathrm{P}_{\mathrm{es}}$ is measured at the same time point; and (4) the $\Delta \mathrm{P}_{\text {es }} / \Delta \mathrm{P}_{\text {aw }}$ ratio is calculated. E: The peak-to-peak method is performed as: the peak negative $P_{\text {aw }}$ and $P_{\text {es }}$ during the occluded inspiratory effort and the peak positive $\mathrm{P}_{\mathrm{aw}}$ and $\mathrm{P}_{\mathrm{es}}$ before the onset of inspiratory effort are localized and measured. $\Delta \mathrm{P}_{\mathrm{es}} / \Delta \mathrm{P}_{\mathrm{aw}}$ ratio is calculated.

tril to a depth of approximate $60 \mathrm{~cm}$. After inflating the balloon with $1.0 \mathrm{~mL}$ air, the gastric position of the balloon was confirmed by a positive or flattened deflection of balloon pressure tracing during inspiration. The catheter was slowly withdrawn until a negative deflection during inspiration appeared in the pressure tracing. The depth of the catheter (from the catheter tip to the nostril) was documented as the pressure inversion point, indicating part of the balloon passing across the gastroesophageal junction. ${ }^{12,13}$ At this balloon position, the cardiac artifact in the $\mathrm{P}_{\mathrm{es}}$ waveform usually presented or abruptly increased. Then the catheter was withdrawn for a further $10 \mathrm{~cm}$, and the balloon volume was re-checked.

We performed the Baydur dynamic occlusion test by an end-expiratory hold to induce 3 inspiratory efforts. ${ }^{3}$ The slope method (as detailed below) was used for the occlusion test measurement at the bedside. Catheter depth was adjusted once for $1.0 \mathrm{~cm}$ and the occlusion test was repeated, until the all 3 slopes were within the range of
$0.8-1.2$, indicating the proper balloon position. ${ }^{2}$ Balloon position adjustment was repeated after change of subject's body position or mechanical ventilation settings.

\section{Off-Line Analysis: Reference Method for Occlusion Test Measurement}

One trained investigator (GQC) inspected the ICU-Lab data for off-line analysis. Special attention was paid to flow tracing to exclude airway leaks. During the inspiratory effort against airway occlusion, the fluctuation of flow within $\pm 30 \mathrm{~mL} / \mathrm{s}$ was allowed because of the slight change in lung volume. Data containing flow, $\mathrm{P}_{\mathrm{aw}}$, and $\mathrm{P}_{\mathrm{es}}$ in each occlusion test with 3 inspiratory efforts were saved as an epoch (Fig. 1A). The first, second, and third inspiratory efforts were marked. Cardiac artifacts on $\mathrm{P}_{\mathrm{es}}$ tracing were measured as the difference in the minimal and the maximal values in an entire cardiac cycle at the end-expiration during normal pressure support ventilation. ${ }^{7}$ 
Linear regression analysis between $\Delta \mathrm{P}_{\mathrm{es}}$ and $\Delta \mathrm{P}_{\mathrm{aw}}$ during the inspiratory effort was performed. The start and the end of an inspiratory effort were identified at the same stage of cardiac pulsation (arrows in Fig. 1A). $\mathrm{P}_{\mathrm{aw}}$ and $\mathrm{P}_{\mathrm{es}}$ data within this range were used in linear regression (Fig. 1B). $\Delta \mathrm{P}_{\mathrm{es}}$ was plotted against $\Delta \mathrm{P}_{\mathrm{aw}}$, and the slope of the regression line was calculated and used as the reference for occlusion test measurement (Fig. 1C). ${ }^{3}$ The phase lag between $\Delta \mathrm{P}_{\text {es }}$ and $\Delta \mathrm{P}_{\text {aw }}$ (represented as degrees) was calculated as the vertical $\Delta \mathrm{P}_{\mathrm{es}}$ difference at the midpoint of horizontal $\Delta \mathrm{P}_{\mathrm{aw}}$ change dividing the total vertical $\Delta \mathrm{P}_{\mathrm{es}}$ change. ${ }^{3,4}$

We defined the epoch with all slopes of the 3 inspiratory efforts within $0.8-1.2$ as an indicator for the optimal balloon position; otherwise the position was deemed nonoptimal. ${ }^{2}$ Thus all collected epochs were classified into 2 categories, optimal and non-optimal.

\section{Off-Line Analysis: Tested Methods for Occlusion Test Measurement}

Three observers (XH, XMS, and YLY) were trained to perform the $\Delta \mathrm{P}_{\mathrm{es}} / \Delta \mathrm{P}_{\mathrm{aw}}$ ratio measurement by 2 tested methods in collected epochs. Each observer performed the measurements independently twice, at a time interval of 2 weeks. No discussion was allowed among the observers.

The cardiac cycle locating method (Fig. 1D) was performed in 4 steps. First, the length of cardiac cycle was measured by rhythmic pulsation on the $\mathrm{P}_{\mathrm{es}}$ tracing. Next, the time point of the peak negative $\mathrm{P}_{\mathrm{aw}}$ during the occluded inspiratory effort was confirmed, and $\mathrm{P}_{\mathrm{aw}}$ and $\mathrm{P}_{\mathrm{es}}$ values were measured. Third, baseline $\mathrm{P}_{\mathrm{aw}}$ before the inspiratory effort was localized and measured at the point with integers of cardiac cycle prior to the peak negative $\mathrm{P}_{\mathrm{aw}}$ point, and $\mathrm{P}_{\mathrm{es}}$ was measured at the same time point. Finally, the $\Delta \mathrm{P}_{\mathrm{es}} / \Delta \mathrm{P}_{\mathrm{aw}}$ ratio was calculated.

The traditional peak-to-peak method (Fig. 1E) was performed according to previous reports. ${ }^{4}$ The peak negative $\mathrm{P}_{\mathrm{aw}}$ and $\mathrm{P}_{\mathrm{es}}$ during the occluded inspiratory effort and the peak positive $\mathrm{P}_{\mathrm{aw}}$ and $\mathrm{P}_{\mathrm{es}}$ before the onset of inspiratory effort were localized and measured. The $\Delta \mathrm{P}_{\mathrm{es}} / \Delta \mathrm{P}_{\mathrm{aw}}$ ratio was calculated. Measurement was restricted within the airway occlusion, and the onset of occlusion was defined as the point of flow returning to zero (dashed line in Fig. 1A).

\section{Statistical Analysis}

Categorical variables are reported as numbers and percentages. Continuous data are presented as median and interquartile range (IQR). Cardiac artifacts and phase lag were compared between the optimal and the non-optimal epochs with the Mann-Whitney $U$ test. Slope, $\Delta \mathrm{P}_{\mathrm{es}}, \Delta \mathrm{P}_{\mathrm{aw}}$, and phase lag among the 3 consecutive inspiratory efforts were compared using the Kruskal-Wallis $\mathrm{H}$ test followed by pairwise comparison with Bonferroni correction.

For $\Delta \mathrm{P}_{\mathrm{es}} / \Delta \mathrm{P}_{\mathrm{aw}}$ ratio measurements with the cardiac cycle locating method and the peak-to-peak method, interobserver and intra-observer reliability of measurement were analyzed using intraclass correlation coefficient (ICC). While the observers and the data epochs were samples of the population, the ICC model was chosen as 2-way random. ${ }^{14}$ Data from the observer who had the highest intraobserver reliability was used for further agreement analysis.

Agreement of respective tested method (the cardiac cycle locating method and the peak-to-peak method) with the reference (the slope method) was assessed using BlandAltman's limits of agreement analysis. ${ }^{15}$ Bias was defined as the mean of the difference between the tested value and the reference value (tested - reference). Upper and lower limits of agreement were defined as bias $\pm 1.96 \mathrm{SD}$ of the mean bias.

The 2 tested methods were also used to assess the optimal balloon position with the criterion of all 3 ratios within $0.8-1.2$ in each epoch ( 1 occlusion test). ${ }^{2}$ Using the slope as the reference, sensitivity and specificity and the respective 95\% CIs of the 2 tested methods were calculated using standard formulas and compared with McNemar's test. ${ }^{16}$

Analyses were conducted using SPSS V.20.0 (SPSS, Chicago, Illinois). $P$ values $<.05$ were considered statistically significant.

\section{Results}

Sixty-two balloon position adjustments and 100 occlusion tests were performed in 15 subjects, whose baseline characteristics are shown in Table 1. During the balloon withdrawal from the stomach, the median (IQR) depth of pressure inversion point was $49(46-50) \mathrm{cm}$ with a range of $44-54 \mathrm{~cm}$. Off-line inspection found airway leaks in 6 adjustments (14 occlusion tests) and data were excluded (see the supplementary table at http://www.rcjournal.com). In all, 86 epochs containing 258 inspiratory efforts were collected for off-line analysis.

The median (IQR) slope of $\Delta \mathrm{P}_{\mathrm{es}}$ versus $\Delta \mathrm{P}_{\mathrm{aw}}$ plot was $0.85(0.76-0.91)$, with a range of $0.23-1.36$. There were $20(23.3 \%), 11(12.8 \%), 10$ (11.6\%), and $45(52.3 \%)$ epochs with none, 1,2 , and all 3 slopes within $0.8-1.2$, respectively. According to predefined criterion, the optimal $(n=45,52.3 \%)$ and non-optimal epochs $(n=41$, $47.7 \%$ ) were classified (Table 2). The median (IQR) cardiac artifact was $1.3(0.9-1.6) \mathrm{cm} \mathrm{H}_{2} \mathrm{O}$, and no significant difference was found between the optimal and non-optimal epoch $(P=.55)$. Compared with the non-optimal epochs, $\Delta \mathrm{P}_{\mathrm{es}}$ was significantly larger in the optimal epochs (13.0 [10.6-18.6] vs 10.1 [6.8-19.1] $\mathrm{cm} \mathrm{H}_{2} \mathrm{O}, P=.001$ ). 
Table 1. Baseline Characteristics of Subjects at Study Entry

\begin{tabular}{|c|c|}
\hline \multicolumn{2}{|l|}{ Characteristics } \\
\hline Age, y & $53(40-70)[21-74]$ \\
\hline Male, $n(\%)$ & $8(53 \%)$ \\
\hline Height, cm & 165 (163-169) [160-182] \\
\hline Weight, kg & $70(64-78)[60-85]$ \\
\hline BMI, $\mathrm{kg} / \mathrm{m}^{2}$ & $25(23-28)[21-31]$ \\
\hline APACHE II score & $14(12-15)[5-19]$ \\
\hline \multicolumn{2}{|l|}{ Mechanical ventilation settings } \\
\hline $\mathrm{F}_{\mathrm{IO}_{2}}$ & 0.4 \\
\hline PEEP, $\mathrm{cm} \mathrm{H}_{2} \mathrm{O}$ & $5.0(4.5-6.0)[4.0-7.0]$ \\
\hline Pressure support, $\mathrm{cm} \mathrm{H}_{2} \mathrm{O}$ & $8.0(7.5-9.0)[7.0-10.0]$ \\
\hline Frequency, breaths/min & $24(22-27)[18-30]$ \\
\hline Tidal volume, $\mathrm{mL}$ & $399(269-463)[236-570]$ \\
\hline $\mathrm{P}_{\mathrm{aO}_{2}}, \mathrm{~mm} \mathrm{Hg}$ & $109(98-137)[74-175]$ \\
\hline $\mathrm{P}_{\mathrm{aCO}_{2}}, \mathrm{~mm} \mathrm{Hg}$ & $34(29-39)[26-44]$ \\
\hline \multicolumn{2}{|c|}{$\begin{array}{l}\text { Continuous data are presented as median (interquartile range) }[\text { range }] . n=15 \text { subjects. } \\
\text { APACHE }=\text { Acute Physiology and Chronic Health Evaluation } \\
\text { BMI }=\text { body mass index }\end{array}$} \\
\hline
\end{tabular}

Phase lag between $\Delta \mathrm{P}_{\mathrm{es}}$ and $\Delta \mathrm{P}_{\mathrm{aw}}$ was significantly higher in the non-optimal epochs compared with the optimal epochs (4.9 [3.3-6.8] vs 6.1 [4.2-8.4] degree, $P=.002$ ).

For $\Delta \mathrm{P}_{\mathrm{es}} / \Delta \mathrm{P}_{\mathrm{aw}}$ ratio measurement, ICCs $(95 \%$ CIs) of inter-observer reliability were $0.992(0.991-0.994)$ in the cardiac cycle locating method and $0.92(0.90-0.93)$ in the peak-to-peak method (see the supplementary table 2 at http://www.rcjournal.com). All ICCs for intra-observer reliability were $>0.99$ (see the supplementary table 3 at http://www.rcjournal.com). Because observer $\mathrm{C}$ had the highest ICC, values measured by this observer were used in further analysis.

Using the slope as the reference, bias (lower and upper limit of agreement) of $\Delta \mathrm{P}_{\mathrm{es}} / \Delta \mathrm{P}_{\mathrm{aw}}$ ratio measured by the cardiac cycle locating method and the peak-to-peak method was $0.02(-0.13-0.16)$ and $0.06(-0.18-0.31)$, respectively (Fig. 2).

The slope method was also used as the reference in identifying the optimal balloon position. Sensitivity and specificity $(95 \% \mathrm{CI})$ for the cardiac cycle locating method were $0.80(0.65-0.90)$ and $0.85(0.70-0.94)$, respectively (Table 3). Respective sensitivity and specificity for the peak-to-peak method were $0.87(0.73-0.95)$ and 0.63 (0.47-0.77). There was a significant difference in the sensitivity and specificity between the 2 tested methods $(P=.008)$, which was due to the significant difference in assessments in the non-optimal epochs $(P=.02)$ (Table 4).

Phase lag, $\Delta \mathrm{P}_{\mathrm{es}}$, and $\Delta \mathrm{P}_{\mathrm{aw}}$ increased significantly during the second and the third occluded inspiratory efforts compared with the first effort (from $P<.001$ to $P=.046$, Fig. $3)$. No significant difference was found in slope among different inspiratory efforts in the same occlusion test $(P=.75)$.

\section{Discussion}

We introduced a method to minimize the influence of cardiac artifacts on $\mathrm{P}_{\mathrm{es}}$ measurement in the dynamic occlusion test. The cardiac cycle locating method provided reliable and precise determination of the $\Delta \mathrm{P}_{\mathrm{es}} / \Delta \mathrm{P}_{\mathrm{aw}}$ ratio during an occluded inspiratory effort. Compared with conventional peak-to-peak method, our method was more specific in detecting the non-optimal balloon position, which is important during esophageal balloon positioning.

In patients with spontaneous breathing, the occlusion test is used to guide esophageal balloon placement. ${ }^{3,4}$ During an inspiratory effort against airway occlusion, there is no change in lung volume and transpulmonary pressure, and thus the negative deflections of $\mathrm{P}_{\mathrm{aw}}$ and $\mathrm{P}_{\mathrm{es}}$ should be almost identical. When the balloon is located in the esophagus, cardiac artifacts usually appear, which can be used as an indicator for the entrance of the balloon into the esophagus. ${ }^{1,2}$ Meanwhile, cardiac artifacts may also interfere with the $\mathrm{P}_{\mathrm{es}}$ measurement. ${ }^{3-5}$ Because dedicated pressure and flow acquisition systems are not routinely incorporated into electrocardiogram monitoring, it is usually impossible to locate the $\mathrm{P}_{\mathrm{es}}$ tracing at the same phase of cardiac cycle. Some reported mean values of cardiac artifacts in supine position were as high as $3 \mathrm{~cm} \mathrm{H}_{2} \mathrm{O}$, ,7 which might significantly influence the calculation of $\Delta \mathrm{P}_{\mathrm{es}}$

Table 2. Data of Collected Epochs Indicating the Optimal and Non-Optimal Balloon Position

\begin{tabular}{|c|c|c|c|c|}
\hline & $\begin{array}{c}\text { All Epochs } \\
\text { ( } n=86 \text { with } 286 \text { efforts) }\end{array}$ & $\begin{array}{c}\text { Optimal Epochs } \\
\text { ( } n=45 \text { with } 135 \text { efforts) }\end{array}$ & $\begin{array}{c}\text { Non-Optimal Epochs } \\
\text { ( } n=41 \text { with } 123 \text { efforts) }\end{array}$ & $P$ \\
\hline Slope & $0.851(0.76-0.91)[0.23-1.36]$ & $0.89(0.87-0.94)[0.81-1.17]$ & $0.76(0.67-0.81)[0.23-1.36]$ & $<.001$ \\
\hline$\Delta \mathrm{P}_{\mathrm{es}}, \mathrm{cm} \mathrm{H}_{2} \mathrm{O}$ & $11.6(8.7-18.7)[3.6-34.1]$ & $13.0(10.6-18.6)[6.7-34.1]$ & $10.1(6.8-19.1)[3.6-30.7]$ & .001 \\
\hline Cardiac artifact, $\mathrm{cm} \mathrm{H}_{2} \mathrm{O}$ & $1.3(0.9-1.6)[0.1-3.6]$ & $1.2(0.9-1.6)[0.5-3.6]$ & $1.4(0.9-1.8)[0.1-2.8]$ & .55 \\
\hline Phase lag, degree & $5.2(3.5-7.5)[-13.9-24.0]$ & $4.9(3.3-6.8)[-13.9-13.4]$ & $5.7(4.0-7.9)[0.5-24.0]$ & .048 \\
\hline \multicolumn{4}{|c|}{ 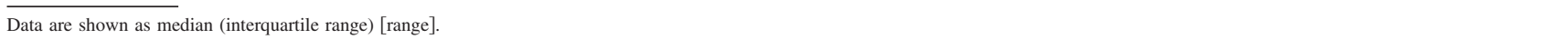 } & \\
\hline
\end{tabular}



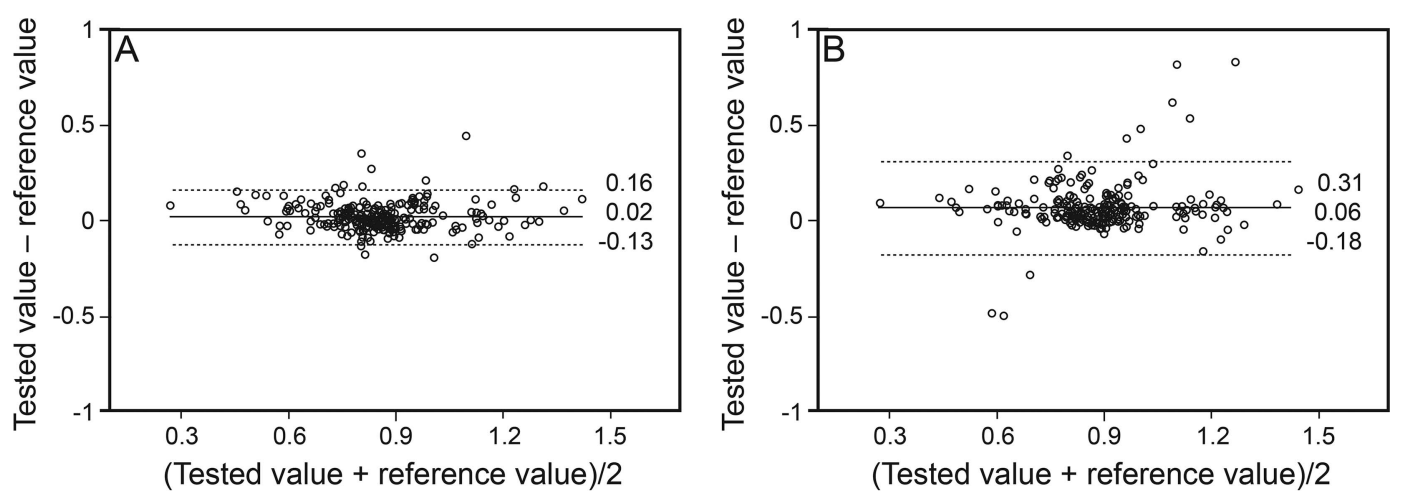

Fig. 2. Bland-Altman's limits of agreement analysis in 2 tested methods versus the reference. Slope of plot between the change in esophageal pressure versus the change in airway pressure is used as the reference. Bias is defined as the mean of the difference between the tested value and the reference value (tested - reference, solid line). Upper and lower limits of agreement are defined as bias \pm 1.96 $\mathrm{SD}$ of the mean bias (dashed line). The cardiac cycle locating method versus the slope method (A). The peak-to-peak method versus the slope method (B).

Table 3. Diagnostic Accuracy Parameters in the 2 Tested Methods for Indicating the Optimal Balloon Position

\begin{tabular}{lcc}
\hline \hline & $\begin{array}{c}\text { Cardiac Cycle } \\
\text { Locating Method }\end{array}$ & Peak-to-Peak Method \\
\hline Sensitivity, \% (95\% CI) & $0.80(0.65-0.90)$ & $0.87(0.73-0.95)$ \\
Specificity, \% (95\% CI) & $0.85(0.70-0.94)$ & $0.63(0.47-0.77)$ \\
PPV, \% (95\% CI) & $0.86(0.71-0.94)$ & $0.77(0.58-0.83)$ \\
NPV, \% (95\% CI) & $0.80(0.64-0.90)$ & $0.81(0.63-0.92)$
\end{tabular}

The slope method was used as the reference.

$\mathrm{PPV}=$ positive predict value

$\mathrm{NPV}=$ negative predict value

during occluded inspiratory effort. For example, in an inspiratory $\mathrm{P}_{\mathrm{es}}$ swing of $-10 \mathrm{~cm} \mathrm{H}_{2} \mathrm{O}$, a cardiac artifact of $3 \mathrm{~cm} \mathrm{H}_{2} \mathrm{O}$ represents $30 \%$ variance in $\Delta \mathrm{P}_{\mathrm{es}}$ calculation if the baseline $\mathrm{P}_{\mathrm{es}}$ is measured at different phases of the cardiac cycle. In our study, the respective median cardiac artifact and $\Delta \mathrm{P}_{\mathrm{es}}$ were 1.3 and $12.7 \mathrm{~cm} \mathrm{H}_{2} \mathrm{O}$ (Table 2 and Fig. $3 \mathrm{C}$ ), which may cause $10 \%$ variance of the calculation. To attenuate the influence of cardiac artifact on $\Delta \mathrm{P}_{\mathrm{es}} / \Delta \mathrm{P}_{\mathrm{aw}}$ ratio measurement, we introduced a method to guarantee the baseline and the peak negative $\mathrm{P}_{\mathrm{es}}$ measurements at the same time point of the cardiac cycle. Using the slope of $\Delta \mathrm{P}_{\mathrm{es}}$ versus $\Delta \mathrm{P}_{\mathrm{aw}}$ plot as the reference, the cardiac cycle locating method provided more accurate and precise measurement of $\Delta \mathrm{P}_{\mathrm{es}} / \Delta \mathrm{P}_{\mathrm{aw}}$ ratio than did the traditional peak-to-peak method (Fig. 2). In an animal experiment, Lanteri et $\mathrm{al}^{6}$ found that the slope and the ratio were comparable for the measurement in occlusion test. Our results confirmed the clinical feasibility for the measurement of the $\Delta \mathrm{P}_{\mathrm{es}} / \Delta \mathrm{P}_{\mathrm{aw}}$ ratio and suggested improvement of accuracy by cardiac cycle locating.

Using a dynamic occlusion test, a $\Delta \mathrm{P}_{\mathrm{es}} / \Delta \mathrm{P}_{\mathrm{aw}}$ ratio of $0.8-1.2$ is recommended to indicate the optimal balloon position. ${ }^{1,2}$ The balloon should be repositioned when the
Table 4. Comparison of Sensitivity and Specificity in the 2 Tested Methods for Indicating Optimal Balloon Position

\begin{tabular}{lrrrr}
\hline \hline & \multicolumn{3}{c}{ Cardiac Cycle Locating } \\
Peak-to-Peak Method & \multicolumn{3}{c}{ Method } & $P$ \\
\cline { 2 - 3 } & + & - & Total & \\
\hline Optimal positions* & 35 & 4 & 39 & .38 \\
$\quad+$ & 1 & 5 & 6 & \\
- & 36 & 9 & 45 & \\
$\quad$ Total & & & & .02 \\
Non-optimal positions* & 4 & 11 & 15 & \\
+ & 2 & 24 & 26 & \\
- & 6 & 35 & 41 & \\
Total & & & & .008 \\
All epochs & 39 & 15 & 54 & \\
+ & 3 & 29 & 32 & \\
- & 42 & 44 & 86 & \\
Total & & &
\end{tabular}

Sensitivity and specificity of the 2 tested methods were compared with McNemar's test.

* Optimal and non-optimal balloon positions were identified using the slope method.

ratio is outside this range. Therefore, it is critical to detect non-optimal balloon positioning during the occlusion test. In this study, we performed off-line analysis of the occlusion test data and defined the optimal epoch. Using the slope method as the reference, the sensitivity and specificity in optimal balloon position assessments were compared between the cardiac cycle locating method and the peak-to-peak method (Tables 3 and 4). In optimal epochs, no significant difference was found between the 2 tested methods (correctly detected $87 \%$ and $80 \%$ of cases, respectively, $P=.38$ ). However, in non-optimal epochs, $85 \%(35 / 41)$ of cases were correctly detected by the cardiac cycle locating method, whereas only $63 \%$ (26/41) of cases were correctly identified by the peak-to-peak method $(P=.02$, Table 4$)$. In other words, more than one third of 

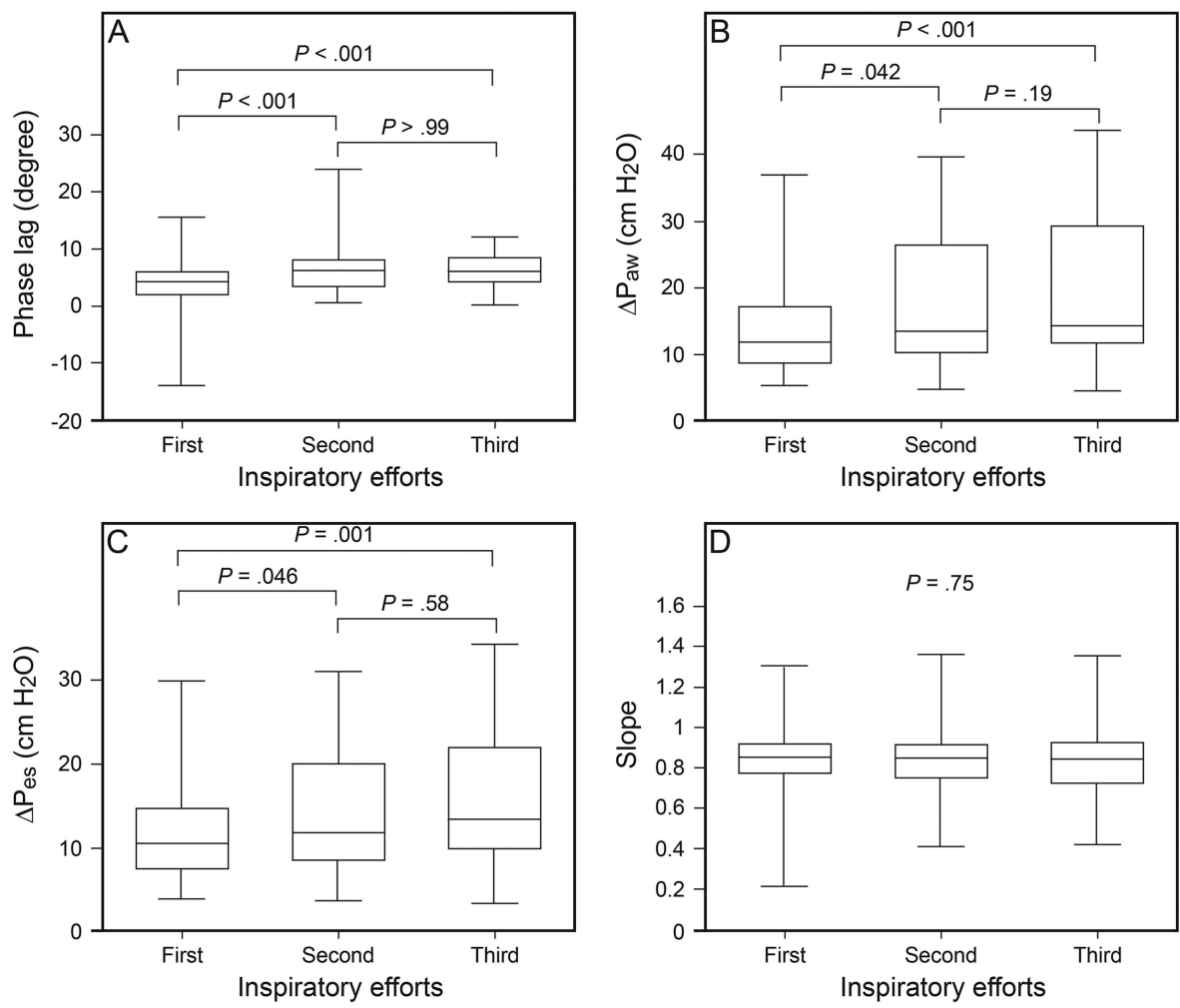

Fig. 3. Phase lag $(A)$, change in airway pressure $\left(\Delta P_{a w}, B\right)$, change in esophageal pressure $\left(\Delta P_{e s}, C\right)$ and slope (D) in the 3 consecutive inspiratory efforts during occlusion test. Pairwise comparisons are shown.

non-optimal positions were evaluated as optimal by the peak-to-peak method. This might result in errors during balloon placement.

It is a prerequisite for the occlusion test that lung volume and transpulmonary pressure do not change during the occluded inspiratory effort. ${ }^{1-4}$ At the bedside performance of the test, clinicians may tend to focus on the $\Delta \mathrm{P}_{\mathrm{es}}$ and $\Delta \mathrm{P}_{\text {aw }}$ measurement and neglect the observation of flow tracing. In our collected occlusion test epochs, offline analysis revealed that airway leak occurred in as many as $14 \%$ of measurements (see the supplementary table 2 at http://www.rcjournal.com). Our data suggest the importance of checking for airway leaks during the bedside performance of the occlusion test.

Three to five occluded inspiratory efforts are required during the occlusion test. ${ }^{1}$ In our collected data, there were $21(24.4 \%)$ epochs in which 1 or 2 slopes were within $0.8-1.2$. In this study, we defined the optimal epoch as having all 3 slope of $\Delta \mathrm{P}_{\mathrm{es}}$ versus $\Delta \mathrm{P}_{\mathrm{aw}}$ plots within the recommended reliable range. ${ }^{2}$ Whether this criteria is suitable for clinical use needs further investigation.

Previous research demonstrated loop formation in the plot of $\Delta \mathrm{P}_{\mathrm{es}}$ against $\Delta \mathrm{P}_{\mathrm{aw}}$ during the occlusion test, in either animal experiments or healthy volunteers. ${ }^{3,4,12}$ Using phase lag to quantify the loop, we found that loop formation was more significant in epochs indicating non- optimal balloon positions than in those indicating optimal positions (Table 2). Additionally, phase lag significantly increased during the 3 consecutive occluded inspiratory efforts (Fig. 3). Similarly, $\Delta \mathrm{P}_{\mathrm{aw}}$ and $\Delta \mathrm{P}_{\mathrm{es}}$ tended to change with the same frequency. We supposed that the pressure transmission across the esophagus might be the reason for loop formation. Distortion of the respiratory system is likely more significant as inspiratory effort increased and thus may attenuate the pressure transmission. Dechman et al ${ }^{12}$ made a similar speculation in their animal experiment. The use of $\mathrm{P}_{\mathrm{es}}$ changes to reflect pleural pressure changes depends on adequate pressure transmission from the pleura to the esophagus. Clinical studies have shown that reliable $\mathrm{P}_{\mathrm{es}}$ measurements can be obtained at a balloon depth of $40 \mathrm{~cm}$ in the majority of patients. ${ }^{17,18}$ However, further investigation is needed to determine whether the balloon position or the patient's body position should be adjusted when a marked loop formation presents during the occlusion test, even though an optimal $\Delta \mathrm{P}_{\mathrm{es}} / \Delta \mathrm{P}_{\mathrm{aw}}$ ratio is obtained.

There are limitations in our study. First, although our proposed method could improve measurement for dynamic occlusion tests, there are several confounding factors for the estimation of pleural pressure from $\mathrm{P}_{\mathrm{es}}$, mainly including the weight of the mediastinal organs, the rib cage, and the abdomen, as well as chest wall compliance and lung 
inflation. ${ }^{19,20}$ It has been reported that the weight of the cardiac vessels may also impose approximately $3 \mathrm{~cm} \mathrm{H}_{2} \mathrm{O}$ on $\mathrm{P}_{\mathrm{es}}$ as a result of changing the body position from upright to supine. ${ }^{21}$ During $\mathrm{P}_{\mathrm{es}}$ monitoring, these factors should be considered. Second, we only enrolled spontaneously breathing subjects and tested the cardiac cycle locating method during the dynamic occlusion test. In subjects who were paralyzed, such as those with ARDS, a positive pressure occlusion test was performed to validate balloon position. ${ }^{22}$ Whether our proposed method can be applied in these patients, especially in the presence of obesity, needs further investigation. Third, the main contraindication of the cardiac cycle locating method is cardiac arrhythmias. Because of the low capability of the peak-to-peak method in detecting non-optimal balloon positioning, we suggest that the slope method be used in arrhythmic patients.

\section{Conclusions}

Our introduced cardiac cycle locating method provides reliable and precise measurement for occlusion tests. More importantly, this method could accurately detect non-optimal balloon position during balloon adjustment and thus decrease the probability of false-optimal balloon placement. Clinicians should pay special attention to flow signals when performing the occlusion test at the bedside.

\section{REFERENCES}

1. Akoumianaki E, Maggiore SM, Valenza F, Bellani G, Jubran A, Loring $\mathrm{SH}$, et al. The application of esophageal pressure measurement in patients with respiratory failure. Am J Respir Crit Care Med 2014;189(5):520-531.

2. Mauri T, Yoshida T, Bellani G, Goligher EC, Carteaux G, Rittayamai $\mathrm{N}$, et al.; on behalf of the PLeUral pressure working Group (PLUG-Acute Respiratory Failure section of the European Society of Intensive Care Medicine). Esophageal and transpulmonary pressure in the clinical setting: meaning, usefulness and perspectives. Intensive Care Med 2016;42(9):1360-1373.

3. Baydur A, Behrakis PK, Zin WA, Jaeger M, Milic-Emili J. A simple method for assessing the validity of the esophageal balloon technique. Am Rev Respir Dis 1982;126(5):788-791.

4. Baydur A, Cha EJ, Sassoon CS. Validation of esophageal balloon technique at different lung volumes and postures. J Appl Physiol 1987;62(1):315-321.

5. Trop D, Peeters R, Van de Woestijne KP. Localization of recording site in the esophagus by means of cardiac artifacts. J Appl Physiol 1970;29(3):283-287.
6. Lanteri CJ, Kano S, Sly PD. Validation of esophageal pressure occlusion test after paralysis. Pediatr Pulmonol 1994;17(1):56-62.

7. Higgs BD, Behrakis PK, Bevan DR, Milic-Emili J. Measurement of pleural pressure with esophageal balloon in anesthetized humans. Anesthesiology 1983;59(4):340-343.

8. Hartford CG, van Schalkwyk JM, Rogers GG, Turner MJ. Primate pleuroesophageal tissue barrier frequency response and esophageal pressure waveform bandwidth in health and acute lung injury. Anesthesiology 2000;92(2):550-558.

9. Schuessler TF, Gottfried SB, Goldberg P, Kearney RE, Bates JH. An adaptive filter to reduce cardiogenic oscillations on esophageal pressure signals. Ann Biomed Eng 1998;26(2):260-267.

10. Cheng YP, Wu HD, Jan GJ, Wang CY. Removal of cardiac beat artifact in esophageal pressure measurement via a modified adaptive noise cancellation scheme. Ann Biomed Eng 2001;29(3):236-243.

11. Mojoli F, Chiumello D, Pozzi M, Algieri I, Bianzina S, Luoni S, et al. Esophageal pressure measurements under different conditions of intrathoracic pressure. An in vitro study of second generation balloon catheters. Minerva Anestesiol 2015;81(8):855-864.

12. Dechman G, Sato J, Bates JH. Factors affecting the accuracy of esophageal balloon measurement of pleural pressure in dogs. J Appl Physiol 1992;72(1):383-388.

13. Chen H, Yang YL, Xu M, Shi ZH, He X, Sun XM, et al. Use of the injection test to indicate the oesophageal balloon position in patients without spontaneous breathing: a clinical feasibility study. J Int Med Res 2017;45(1):320-331.

14. Muller R, Buttner P. A critical discussion of intraclass correlation coefficients. Stat Med 1994;13(23-24):2465-2476.

15. Bland JM, Altman DG. Statistical methods for assessing agreement between two methods of clinical measurement. Lancet 1986;1(8476): 307-310.

16. Kim S, Lee W. Does McNemar's test compare the sensitivities and specificities of two diagnostic tests? Stat Methods Med Res 2017; 26(1):142-154.

17. Talmor D, Sarge T, O’Donnell CR, Ritz R, Malhotra A, Lisbon A, Loring SH. Esophageal and transpulmonary pressures in acute respiratory failure. Crit Care Med 2006;34(5):1389-1394.

18. Talmor D, Sarge T, Malhotra A, O’Donnell CR, Ritz R, Lisbon A, et al. Mechanical ventilation guided by esophageal pressure in acute lung injury. N Engl J Med 2008;359(20):2095-2104.

19. Talmor DS, Fessler HE. Are esophageal pressure measurements important in clinical decision-making in mechanically ventilated patients? Respir Care 2010;55(2):162-174.

20. Hedenstierna G. Esophageal pressure: benefit and limitations. Minerva Anestesiol 2012;78(8):959-966.

21. Washko GR, O'Donnell CR, Loring SH. Volume-related and volume-independent effects of posture on esophageal and transpulmonary pressures in healthy subjects. J Appl Physiol 2006;100(3):753758.

22. Chiumello D, Consonni D, Coppola S, Froio S, Crimella F, Colombo A. The occlusion tests and end-expiratory esophageal pressure: measurements and comparison in controlled and assisted ventilation. Ann Intensive Care 2016;6(1):13.

This article is approved for Continuing Respiratory Care Education credit. For information and to obtain your CRCE

(free to AARC members) visit www.rcjournal.com

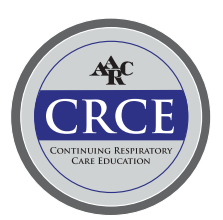

